Document downloaded from:

http://hdl.handle.net/10251/60321

This paper must be cited as:

Broatch Jacobi, JA.; Galindo Lucas, J.; Navarro García, R.; Garcia Tiscar, J. (2014). Methodology for experimental validation of a CFD model for predicting noise generation in centrifugal compressors. International Journal of Heat and Fluid Flow. 50:134-144. doi:10.1016/j.ijheatfluidflow.2014.06.006.

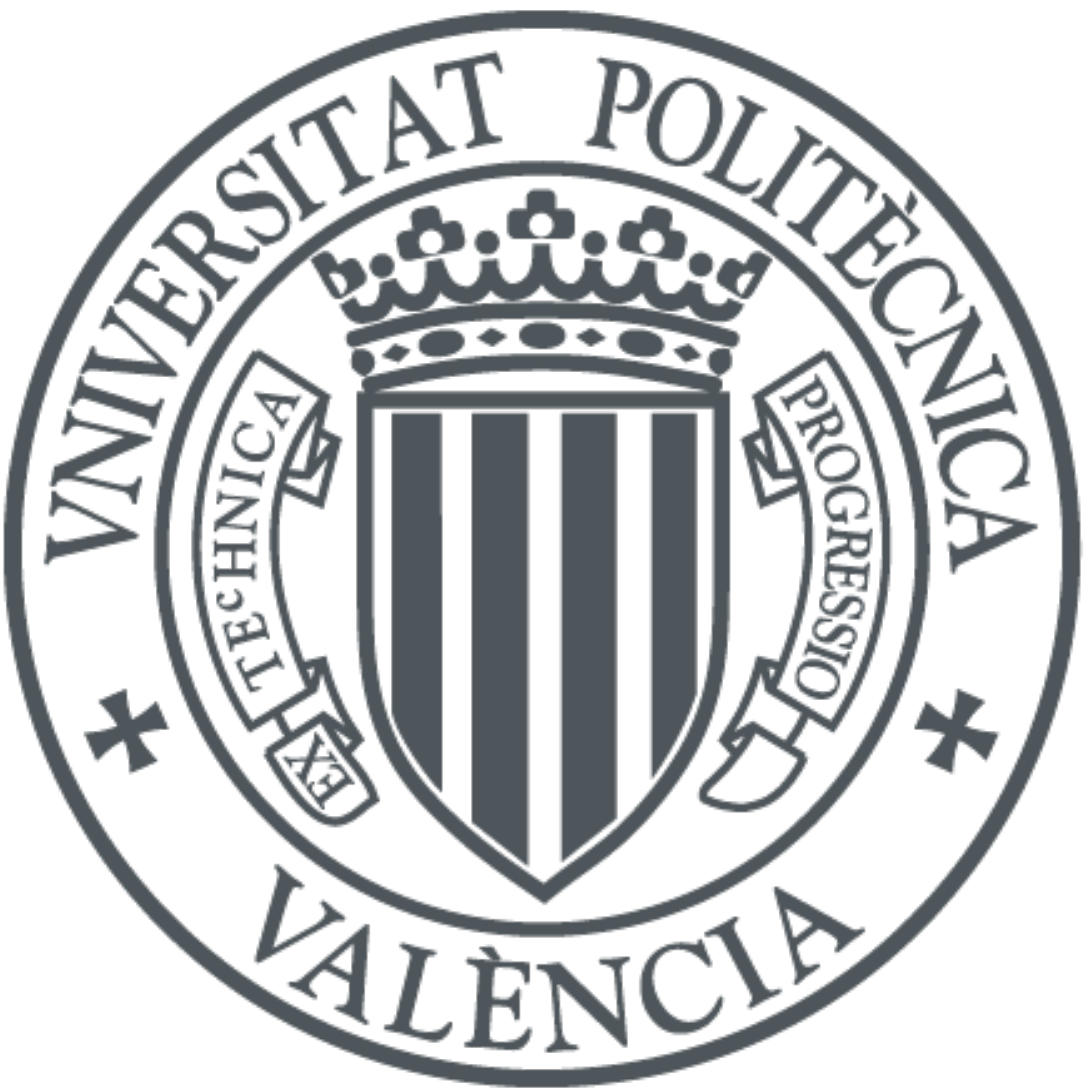

The final publication is available at

http://dx.doi.org/10.1016/j.ijheatfluidflow.2014.06.006

Copyright Elsevier

Additional Information 


\section{NOTICE:}

this is the author's version of a work that was accepted for publication in International Journal of Heat and Fluid Flow. Changes resulting from the publishing process, such as peer review, editing, corrections, structural formatting, and other quality control mechanisms may not be reflected in this document. Changes may have been made to this work since it was submitted for publication. A definitive version was subsequently published as:

A. Broatch, J. Galindo, R. Navarro, J. García-Tíscar, Methodology for experimental validation of a CFD model for predicting noise generation in centrifugal compressors, International Journal of Heat and Fluid Flow 50 (2014) 134-144. doi:10.1016/j.ijheatfluidflow.2014.06.006 


\title{
Methodology for experimental validation of a CFD model for predicting noise generation in centrifugal compressors
}

\author{
A. Broatch, J. Galindo, R. Navarro*, J. García-Tíscar \\ CMT - Motores Térmicos, Universitat Politècnica de València \\ Camino de Vera, 46022 Valencia
}

\begin{abstract}
Centrifugal compressors working in the surge side of the map generate a broadband noise in the range of 1 to $3 \mathrm{kHz}$, named as whoosh noise. This noise is perceived at strongly downsized engines operating at particular conditions (full load, tip-in and tip-out maneuvers). A 3-dimensional CFD model of a centrifugal compressor is built to analyze fluid phenomena related to whoosh noise. A detached eddy simulation is performed with the compressor operating at the peak pressure point of $160 \mathrm{krpm}$. A steady flow rig mounted on an anechoic chamber is used to obtain experimental measurements as a means of validation for the numerical model. In-duct pressure signals are obtained in addition to standard averaged global variables. The numerical simulation provides global variables showing excellent agreement with experimental measurements. Pressure spectra comparison is performed to assess noise prediction capability of numerical model. The influence of the type and position of the virtual pressure probes is evaluated. Pressure decomposition is required by the simulations to obtain meaningful spectra. Different techniques for obtaining pressure components are analyzed. At the simulated conditions, a broadband noise in 1-3 kHz frequency band is detected in the experimental measurements. This whoosh noise is also captured by the numerical model.
\end{abstract}

Keywords: Aeroacoustics, Turbocharger, Whoosh noise, Beamforming,

\footnotetext{
*Corresponding author. Tel.: +34 963877650

Email addresses: abroatch@mot.upv.es (A. Broatch), galindo@mot.upv.es (J. Galindo), ronagar1@mot.upv.es (R. Navarro), jorgarti@mot.upv.es (J. García-Tíscar)
} 
Method of Characteristics

\section{Introduction}

In the automotive industry, the development of strongly downsized engines with increased low speed torque has raised turbocharger airborne noise [1], because the compressor working line is shifted towards surge region [2].

In particular, a broadband noise is detected during some engine conditions (full load, tip-in and tip-out maneuvers). Researchers refer to this noise with terms such as hiss [3] or whoosh [1, 2]. Whoosh noise is commonly described as a broadband noise either in the $1 \mathrm{kHz}-3 \mathrm{kHz}$ band [1, 4] or in frequencies ranging from $4 \mathrm{kHz}$ to $12 \mathrm{kHz}$ [2, 5]. This noise is more audible when the compressor is working in near-surge conditions [1].

Raitor and Neise [6] conducted an experimental study to describe the sound generation mechanisms of centrifugal compressors. For low compressor speeds, corresponding to subsonic flow conditions, radial compressor noise is dominated by tip clearance noise (TCN). TCN is a narrow-band noise observed at frequencies about half the blade passing frequency (BPF), which increase with speed. For their operating conditions, TCN ranged from 3 to 4 $\mathrm{kHz}$. The authors concluded that TCN is due to the secondary flow through the gap between rotor blade tips and the casing wall. It is not clear if TCN can be related to whoosh noise.

Evans and Ward [1] studied turbocharger generated whoosh noise. The noise transmission path was analyzed and the compressor outlet hose was found to be the main source of radiated noise. Increasing hose thickness or mass gave significant reductions in the radiation of whoosh noise. Broadband resonators also provided similar benefits.

Teng and Homco [2] investigated radiated noise on a powertrain dyno in a semi-anechoic cell measured at full load conditions. Different countermeasures to reduce whoosh noise were evaluated on the dyno, i. e., the use of compressors with different trim or pre-whirl. The primary focus of this measures was on reducing the excitation source by improving the compressor surge margin. The effects of various resonators in the path at different locations were also studied.

Sevginer et al. [7] conducted a experimental study in order to detect and eliminate two types of noise generated by the turbocharger: whoosh and blow noise. Measurements were performed in a test vehicle and in an engine 
test rig. A broad-band resonator was succesfully used to diminish whoosh noise in the compressor outlet.

Some of the presented works [1, 2, 3, 7] postulate turbulence generation as the source of whoosh noise, but no evidence supporting this assertion is provided. Numerical simulations of centrifugal compressor flow would be helpful to gain insight of the whoosh noise phenomenon.

Mendonça et al. 8] conducted a numerical study regarding flow-induced aeroacoustics of an automotive radial compressor. SPL spectra were obtained for points in both inlet and outlet ducts, showing a narrow band noise at a frequency about $70 \%$ of rotational speed, which corresponds to $2.5 \mathrm{kHz}$ for the investigated compressor speed. A spiral mode propagating upstream from the compressor impeller was detected for the narrow band noise aforementioned. Leading-edge separation and stalled passages were found, along with a low momentum region that rotates at a slower speed than the wheel. Rotating stall was thus regarded as the source of the narrow band noise. Tip leakage was considered to be the mechanism that allows the stalled passages to recover by pushing the low momentum region to the rotation-trailing passage. Mendonça et al. did not include experimental measurements in their work. Therefore, it can not be confirmed if the narrow band attributed to rotating stall corresponds to whoosh noise.

Karim et al. 9] identified the bad incidence angle that low flow rates present at the leading edge of the compressor blades as the reason of whoosh noise. Large Eddy Simulations were performed in two different operating conditions with 5 different inlet configurations (using swirl vanes, short and large steps and combinations of these elements). SPL integrated over 6-12 $\mathrm{kHz}$ obtained at the compressor inlet was compared for the different inlet configurations. The combination of a large step with swirl vanes provided less SPL for both operating conditions. Experimental measurements with short and large step were performed in a powertrain dynamometer semianechoic cell. SPL integrated between 4 and $12.7 \mathrm{kHz}$ using radiated noise measurements showed the superior performance of the large step over the small step.

Lee et al. [10] conducted an experimental and numerical study of the noise radiated by a turbocharger. A turbocharger test rig was mounted on an anechoic chamber, and noise was measured with a freefield microphone placed at $0.8 \mathrm{~m}$ from the inlet of the compressor. Noise spectra for three rotational speeds were obtained. A narrow band was found at a frequency about 3 times the rotational speed, corresponding to frequencies between 3.5 
and $5.5 \mathrm{kHz}$. This was the dominant frequency in most configurations, and was attributed to the experimental setup. Besides, CFD transient calculations of the turbocharger compressor were performed. Unsteady data for one revolution was extended by repetition for acoustic analysis. Acoustic analogy was used to obtain noise spectra. The numerical approach overpredicted the measured noise spectrum and was not able to detect the narrow band component.

Karim et al. 9] and Lee et al. [10] combined numerical simulations and experiments to study compressor noise radiation. However, Karim et al. only used measurements to confirm the reduction of overall radiated whoosh noise level when increasing the length of the leading edge step. Lee et al. compared measured and numerical prediction of compressor noise spectrum, showing a lack of agreement. Probably, repetition of one revolution data for acoustic analogy led to poor spectrum estimation.

Literature review shows that unsteady numerical simulations are a good tool to analyze compressor aeroacoustics. Moreover, flow decomposition methods such as Dynamic Mode Decomposition (DMD) or Proper Orthogonal Decomposition (POD) can be used to identify flow structures attributed to certain frequencies. Researchers such as Alenius [11, Sakowitz et al. [12] and Kalpakli et al. [13] have shown the validity of these techniques to extract coherent flow structures in engine-like flow conditions. In any case, experimental measurements are required to assess the ability of these simulations to capture compressor noise generation. However, the computational cost of modeling the whole turbocharger test rig is increased because of the existence of long ducts [14. It is a common approach to set virtual pipes' length so as to allow the development of the flow from boundary conditions instead of using the actual duct length. The methodology to compare numerical and experimental results is thus a key point to obtain a meaningful comparison.

In this paper, the validation of a 3D CFD model of a centrifugal compressor with the objective of noise prediction against experimental measurements is described. In section 2, the experimental setup and the procedure for the acoustic characterization of the turbocharger is described, and the compressor map is obtained. The numerical model is defined in section 3 and the methodology to compare the simulation against experimental measurements is developed in section 4. Finally, conclusions and ongoing research using the 3D CFD model are commented in section 5 . 


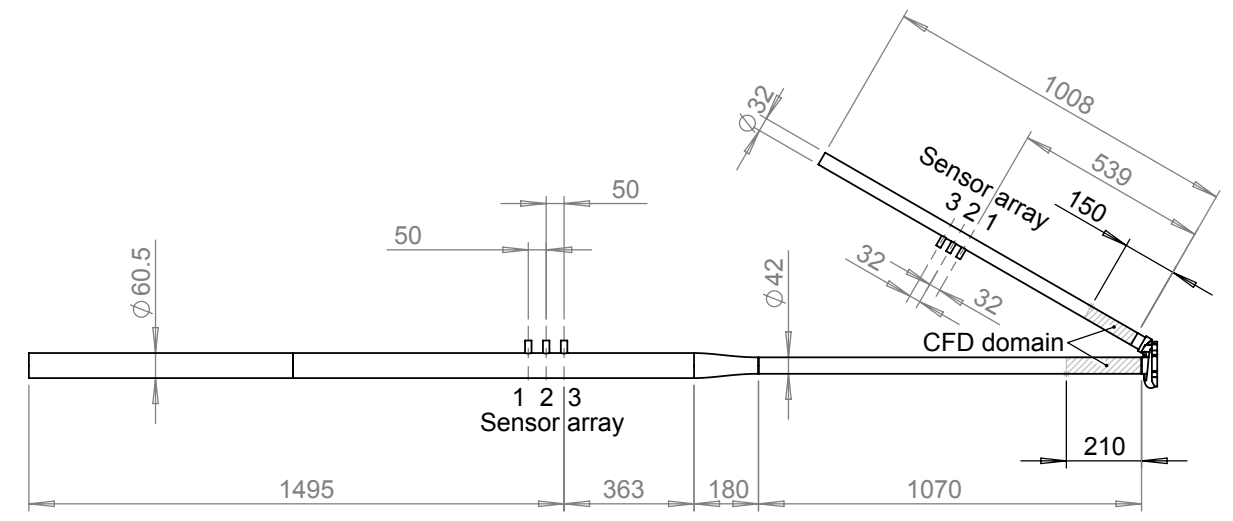

Figure 1: Compressor piping layout, highlighting the CFD domain and the sensor arrays

\section{Experimental methodology}

\subsection{Turbocharger noise measurements}

Various methods for the acoustical analysis of radial turbochargers are available in the literature. The most commonly considered measurements are external radiated noise [1, 2], orifice noise [5, 10], and internal (in-duct) flow noise [1, 3, 15].

External and orifice noises are usually measured using free field microphones or portable spectrum analyzers [5]. For in-duct measurements, piezoelectric pressure transducers are the preferred choice.

Some authors [5, 10] rely on single sensor measurements for either external and internal measurements, while others prefer sophisticated methods involving more sensors in order to obtain spatially averaged noise on the external case [1, 2], or to allow the use of wave decomposition techniques on the internal case [3, 15].

\subsection{Selected methods}

Since the present study is devoted to the comparison between experimental measurements and a CFD model, and since the latter only simulates the in-duct flow (and not chamber ambient through which sound radiation could be resolved), only in-duct experimental measurements will be covered in this paper.

Both single sensor and multi sensor methods were considered, in order to evaluate their adequacy when comparing experimental data with numerical 
results. Single sensor methods were applied using one sensor from a multi sensor array.

Two linear arrays of three piezoelectric sensors each were designed in accordance with Piñero et al. [16], who presented a beamforming-based signal analysis method that allows the decomposition of the pressure signal into a forward-traveling wave $p_{\text {forw }}(t)$ and a backward-traveling wave $p_{\text {back }}(t)$.

Power spectral density (PSD) of these pressure components will be used in section 4 as a means of validation for numerical predictions. However, the decomposition method relies on the plane wave assumption, so it is limited to low and medium frequencies. Moreover, the spacing of the sensors defines a Nyquist-type upper frequency limit [17] which must be considered to avoid aliasing problems. These constraints will also be covered in section 4 .

\subsection{Experimental setup}

The experimental work required in this investigation was carried out at a facility of CMT - Motores Térmicos that hosts a large anechoic chamber and a Diesel-powered gas stand.

In order to properly isolate its acoustical properties, the turbocharger was installed at the center of the anechoic chamber, being powered from the outside by the nearby gas stand and fed with oil from a pump located in the adjacent utility room.

For this investigation, the compressor was fitted with idealized inlet and outlet geometry consisting of long straight steel pipes, in order to have the simplest geometry for CFD modeling. A schematic of this arrangement can be seen in Fig. 1, in which piezoelectric sensor arrays are depicted and the domain modeled using CFD is highlighted.

The gas stand used to power the turbocharger has been previously described by Galindo et al. [18]. Its core is a $10 \mathrm{~L}$ Diesel engine which powers a $150 \mathrm{~kW}$ screw compressor.

Compressed air passes through a water intercooler and a settling tank to ensure cold and steady conditions and then is split to both supercharge the engine and power the turbine of the turbocharger group in the anechoic chamber, which discharges at low speed through an insulated reservoir. The centrifugal compressor studied in this paper is driven by the aforementioned turbine. The compressor takes its air from the chamber ambient and discharges to the adjacent auxiliary room.

Two separate data acquisition systems have been used to perform the experimental measurements. In the first place, a custom-built control system 
monitors averaged pressures, temperatures, mass flows and engine speeds. It is also used to control the valves that set the compressor operating point, oil heating, etc. A 5 seconds average of every sensor is recorded for each data point captured.

Also shown in the schematic are two arrays of piezoelectric pressure sensors mounted on both the inlet and outlet pipes and sampled at $100 \mathrm{kHz}$ during 1 second for each data point, using the second acquisition system, a Yokogawa digital oscilloscope. These arrays enable the use of the wave decomposition technique mentioned in section 2.2 .

\subsection{Compressor map}

Several data points were captured using this setup. The procedure involved a step by step reduction in air mass flow, attained with the operation of the back pressure valve, while a certain turbo speed was maintained regulating the operating pressure of the screw compressor at the gas stand.

For each turbo speed isoline, data was captured between fully opened back pressure valve conditions and the start of deep surge. This process was repeated for $80,100,120,140,160,170$ and $180 \mathrm{krpm}$, although only one data point was captured at the last speed, due to operational limitations of the facility.

To account for the variation in ambient conditions during the measurements, air mass flow and turbocharger speed were corrected to Standard Day conditionst.

$$
N^{*}=N \sqrt{\frac{T_{r e f}}{T_{i n, 0}}} \quad \dot{m}^{*}=\dot{m}\left(\frac{p_{r e f}}{p_{i n, 0}}\right) \sqrt{\frac{T_{i n, 0}}{T_{r e f}}}
$$

Turbocharger speed $N$, air mass flow $\dot{m}$, total inlet pressure $P_{i n, 0}$ and temperature $T_{i n, 0}$ were computed using averaged sensor data from the lab control system.

For each operating condition, the total-to-total pressure ratio

$$
\Pi_{t, t}=\frac{p_{\text {out }, 0}}{p_{\text {in }, 0}}
$$

was obtained from the same system, and plotted against the corrected air mass flow $\dot{m}^{*}$, as shown in Fig. 2, commonly referred to as compressor map.

\footnotetext{
${ }^{1} T_{\text {ref }}=288.15 \mathrm{~K}$ and $P_{\text {ref }}=101325 \mathrm{~Pa}$
} 


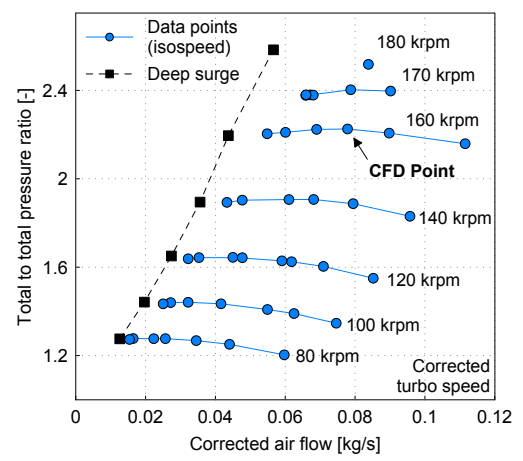

Figure 2: Measured compressor map showing experimentally captured data points, deep surge line and the numerically simulated operating condition

In this kind of map, the leftmost points of each speed line conform the socalled deep surge line. Although this limit is usually regarded as a property of the compressor, previous experience [19, 20, 21] shows that compressor inlet conditions have a great influence on surge onset.

These deep surge points for the selected compressor and inlet geometry were measured in accordance with the method developed by Galindo et al. 22] (see specially Fig. 6 of the paper) using the instantaneous pressure recording of the first array sensor. The measurements were carried out in a separate non-anechoic facility capable of higher power delivery to the turbine at all speeds.

In order to investigate whoosh noise generation, a detached eddy simulation is performed in the point corresponding with highest pressure ratio at $160 \mathrm{krpm}$, marked in Fig. 2. In the following sections, the CFD model noise prediction capabilities will be assessed by the experimental measurements already presented.

\section{Numerical Model}

A numerical model of the compressor was built using Star-CCM+ [23]. The geometry of the impeller, vaneless diffuser and volute were obtained by reverse engineering. The compressor wheel consist of 6 full blades and 6 splitter blades. The tip clearance and the narrow gap between the rear part of the impeller and the backplate are included in the model. Straight 
inlet and outlet ducts were created extruding 5 diameters the corresponding cross section. These ducts are considered instead of the actual ones forming the turbocharger test rig (see Fig. 1) because it is estimated that the computational effort would increase about 10 times if the whole rig was to be simulated. The modeled domain is depicted in Fig. 3.

The numerical configuration is based on the previous work by Mendonça et al. [8]. The mesh consists of 9.5 million polyhedral cells. Figure 4 shows the rotor mesh, which was built so as to obtain $y^{+}$values close to the unity at the impeller. Blade tip clearance and backplate region can be observed in Fig. 4 .

The segregated solver was used to perform a detached-eddy simulation with a SST $k-\omega$ turbulence model, "which functions as a sub-grid-scale model in regions where the grid density is fine enough for a large-eddy simulation, and as a Reynolds-averaged model in regions where it is not" [24]. Particularly, IDDES [25] is used, which combines WMLES and DDES hybrid RANS-LES approaches. The pressure was set at the outlet as a boundary condition and, unlike Mendonça et al. [8], a mass flow boundary condition was used at the inlet.

A transient, rigid body motion simulation was carried out using the operating conditions described in Table 1 to model the point marked in Fig. 2. Mass flow rate is 1.8 times the one corresponding to surge at this compressor speed. The time step is $1.046 \cdot 10^{-6} \mathrm{~s}$, so that the impeller mesh turns $1^{\circ}$ per time step at the selected operating condition. Heat transfer with the

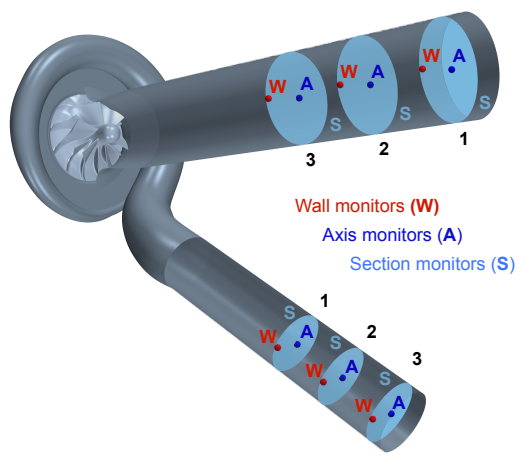

Figure 3: CFD Modeled domain, showing the two arrays of virtual probes located at the inlet and outlet ducts 


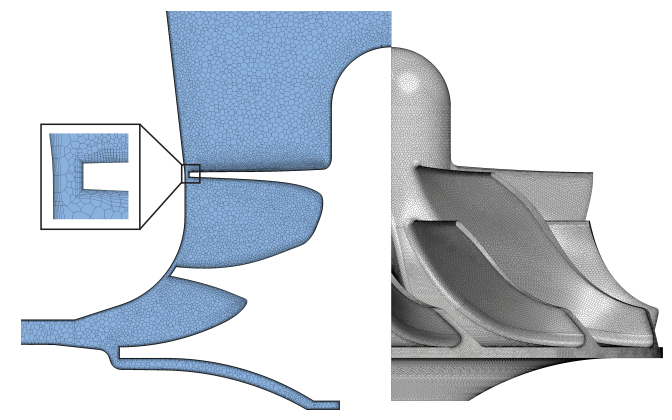

Figure 4: Rotor region mesh: cross-section (left) and impeller (right), showing a detail view of tip clearance and the boundary layer mesh inflation

Table 1: Simulation operating conditions

\begin{aligned} & \hline Inlet boundary condition $=0.077 \mathrm{~kg} / \mathrm{s}, T_{0}=293 \mathrm{~K} \\ &$ Outlet boundary condition $p=223000 \mathrm{~Pa} \\ &$ Rotational speed $N=159000 \mathrm{rpm} \\ &$\hline\end{aligned}

surroundings is neglected by considering adiabatic walls. Serrano et al. [26] showed that heat transfer is only a relevant fraction of the power absorbed by the compressor for low to medium loads. Therefore, prediction of compressor outlet temperature [27] or measurement of isentropic efficiency [28] are not influenced by heat transfer for high compressor speeds, such as the one studied in this paper.

\section{Validation methodology}

\subsection{Global variables}

In order to validate the numerical model described in section 3 , the results obtained by the model are compared with the experimental measurements corresponding to the simulated operating condition. Specific work and isen- 
tropic efficiency

$$
\begin{aligned}
W_{u} & =\frac{\dot{W}}{\dot{m}}=\frac{\frac{2 \pi N(r p m)}{60} \tau}{\dot{m}}=c_{p}\left(T_{\text {out }, 0}-T_{\text {in }, 0}\right) \\
\eta_{s} & =\frac{\dot{W}_{s}}{\dot{W}}=\frac{T_{\text {in }, 0}\left(\Pi_{t, t}^{\frac{\gamma-1}{\gamma}}-1\right)}{T_{\text {out }, 0}-T_{\text {in }, 0}}
\end{aligned}
$$

are considered in addition to compressor map variables (Eqs. 11 and 2) to assess the ability of the model to predict the overall behavior of the turbomachine. These variables are time averaged in both experimental measurements and transient simulations.

The comparison between measured and predicted global variables is made in Table 2, in which relative error for a generic variable $\phi$ is defined as

$$
\epsilon_{R}(\%)=\frac{\left|\phi_{C F D}-\phi_{e x p}\right|}{\phi_{\exp }} .
$$

Table 2: Compressor global variables measured in the experimental test rig and predicted by the numerical model.

\begin{tabular}{cccc}
\hline & $\Pi_{t, t}[-]$ & $W_{u}\left[\mathrm{~kJ} \cdot \mathrm{kg}^{-1}\right]$ & $\eta_{s}[\%]$ \\
\hline Exp. & 2.240 & 112.27 & 67.82 \\
$\mathrm{CFD}$ & 2.221 & 111.32 & 67.62 \\
\hline$\epsilon_{R}(\%)$ & 0.87 & 0.84 & 0.30 \\
\hline
\end{tabular}

Since inlet mass flow and stagnation temperature and outlet pressure are imposed in the simulation (see Table 1), the calculation of inlet stagnation pressure determines the error in prediction of pressure ratio and corrected mass flow rate. Similarly, torque (or outlet stagnation temperature) computation dictates specific work estimation and efficiency is affected by both inlet total pressure and outlet stagnation temperature.

Although the agreement in terms of global variables is excellent (relative errors do not exceed $1 \%$ in Table 2), further validation is required to accept the results provided by the CFD model as a means to study the noise generated by the flow field patterns inside the compressor. For instance, Hemidi 
et al. [29, 30] showed that, for a supersonic air ejector, validation based on global variables does not guarantee proper prediction of local flow features.

Flow measurements inside the device are common for large turbomachinery (e.g., Ubaldi et al. [31]), but for automotive turbochargers it is quite difficult to place sensors due to the small size of the device.

Hellström et al. [32] performed PIV measurements of the flow upstream the inducer of a centrifugal compressor with ported shroud at near surge conditions, being able to capture the main flow structures at this location with a large eddy simulation. In a continuation of this work, Semlitsch et al. [33] confirmed LES potential by showing good quantitative agreement with PIV velocity data at different cross-sections upstream of the impeller, for both stable and near-surge operating conditions. Even though recent studies have been able to obtain PIV-based aeroacoustic predictions, mostly for external flow applications [34, a direct comparison between numerical and experimental unsteady pressure measurements is preferred in this paper.

Choi et al. [35] obtained experimental pressure traces with Kulite sensors to validate a simulation of rotating stall in a transonic fan, placing the pressure probes close to the fan blades. However (instrumentation difficulties aside) Choi et al. compared rotational speed of stall cells whereas this paper
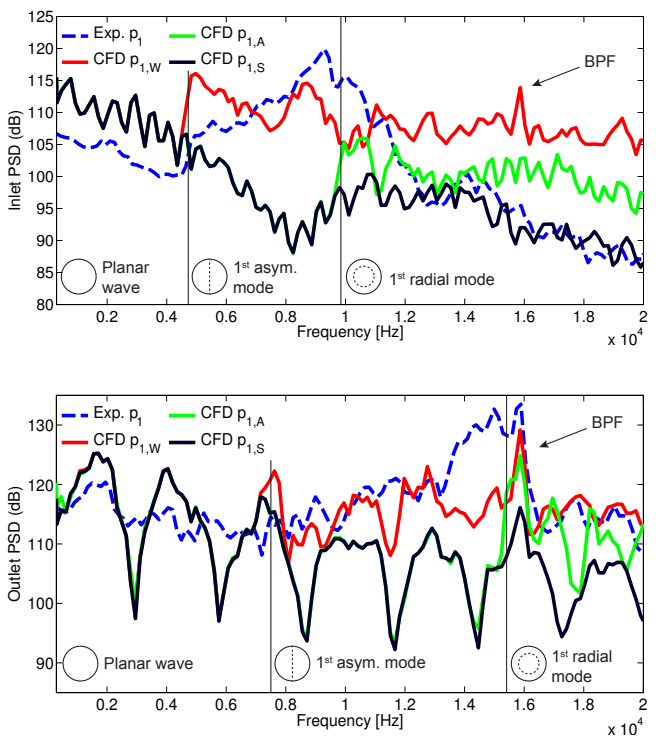

Figure 5: PSD of experimental and numerical probes at inlet (top) and outlet (bottom), including the appearance of the first asymmetric and the first circular symmetric modes. 
is interested in the evaluation of whoosh noise prediction, which is a problem for car manufactures due to excitation and subsequent radiation of inlet and outlet hoses [1].

Therefore, the Power Spectral Density of the pressure signals at both the inlet and the outlet ducts will be used as the tool to assess the quality of the noise prediction delivered by the model.

\subsection{Pressure spectra}

As explained in section 2.3, an array of three piezoelectric sensors was placed at both the inlet and the outlet ducts of the actual turbocharger. Unfortunately, the computational cost of simulating the whole system is prohibitive, and the array of three virtual probes was placed at the extruded ducts of the model, following the recommendations of Piñero et al. [16] to select their axial location. Three different monitors were investigated: two point monitors located respectively at the duct axis and next to the wall and a cross-section monitor. A sketch of the monitors is depicted in Fig. 3 .

PSD can be computed from the raw pressure signal registered by a piezoelectric sensor/monitor. Experimental signals were sampled during $1 \mathrm{~s}$, whereas numerical monitors were stored for over $60 \mathrm{~ms}$ (corresponding to 160 impeller revolutions) after reaching a steady state in terms of global variables. Welch's overlapped segmented average [36] is used to estimate the PSD. Blocks with 50\% overlap are tapered using Hamming function, although no differences were found between most common windowing functions. The number of blocks is selected so as to obtain the frequency resolution closest to $150 \mathrm{~Hz}$.

In Fig. 5, the PSD at the inlet and outlet ducts is presented for the first probe of each array. Experimental spectrum at inlet shows an initial decay until $5 \mathrm{kHz}$ and a broadband elevation from $5 \mathrm{Khz}$ to $12 \mathrm{kHz}$. From $12 \mathrm{kHz}$ onwards, the PSD decreases. The most relevant features for the experimental spectrum at the outlet duct are a broadband elevation from 13 $\mathrm{kHz}$ until $16 \mathrm{kHz}$ and the tone corresponding to the BPF (about $16 \mathrm{kHz}$ for this compressor speed).

In the CFD calculation, the three types of monitor are investigated. At the inlet, all numerical monitors at same axial position provide identical results up to $4.4 \mathrm{kHz}$, whereas the threshold increases to $7 \mathrm{kHz}$ for the outlet. Similarly, axis and cross-section spectra are coincident until $9.5 \mathrm{kHz}$ at the inlet pipe and 15.3 at the outlet. 


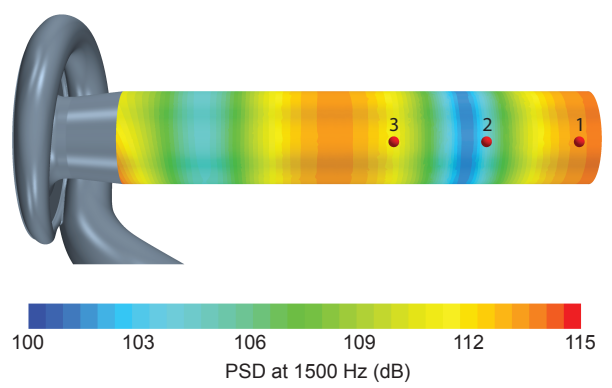

Figure 6: PSD level at $1.5 \mathrm{kHz}$ for inlet duct wall elements, including array of wall (W) point monitors

According to Eriksson [37], first asymmetric mode and first radial mode start propagating at cut-off frequencies of:

$$
f_{c, a}=1.84 \frac{a}{\pi D}\left(1-M^{2}\right)^{1 / 2} \quad f_{c, r}=3.83 \frac{a}{\pi D}\left(1-M^{2}\right)^{1 / 2}
$$

These expression predicts values of $4.7 \mathrm{kHz}$ and $7.4 \mathrm{kHz}$ for inlet and outlet ducts, respectively, for the asymmetric mode. The radial mode starts propagating at $9.7 \mathrm{kHz}$ at the inlet duct and at $15.4 \mathrm{kHz}$ at the outlet. The behavior of the different types of monitor in Fig. 5 is thus caused by the onset of higher order modes.

The agreement between experimental and numerical spectra depicted in Fig. 5 is not good. The decay of the inlet spectrum until $5 \mathrm{kHz}$ and the BPF tone are features captured by the CFD simulation, but numerical spectra presents ripples (see outlet spectrum for cross-section monitor in Fig. 5) that are not present in the measurements. Moreover, if the PSD of same type of monitor at different axial positions are plotted (not shown here), these ripples exists at other frequencies, which could be attributed to standing waves acting at certain frequencies. Contours of PSD at duct walls reveal standing waves at several frequencies, such as at $1500 \mathrm{~Hz}$ for the inlet duct (Fig. 6).

Standing waves are relevant in the numerical model because the length of straight ducts tunes frequencies of $1 \mathrm{kHz}$ and above. If the whole turbocharger rig depicted in Fig. 1 had been simulated, standing waves would have been removed from the frequency range of interest. However, simulating such long ducts would increase the overall computational effort, as stated in Section 1 . 
Non-reflecting boundary conditions (NRBC) could have alleviated this issue, but they are not used in the computation. NRBC represent the behavior of an infinite duct, which is not exactly the case of the experimental rig, but it is more realistic than fixing the pressure at a short length after the compressor, as done in the present simulation. In this way, NRBC should erase the spurious tuning effect on certain frequencies that presents the selected duct length.

Mendonça et al. 8 8 used a NRBC at the inlet while a pressure boundary condition is applied at the outlet. Torregrosa et al. [? ] and Galindo et al. 14] showed that Riemann-based NRBCs, such as the ones existing in StarCCM+ or Ansys-Fluent, do not reproduce truthfully the behavior of an infinite duct for non-homentropic flows. In any case, NRBC were not used in the present paper for different reasons. When a NRBC was set at the outlet, the operating condition instantaneously changed, eventually reaching compressor surge. Creating a longer outlet duct and considering radial equilibrium in the outlet $\mathrm{NRBC}$ [14] may increase the stability of the simulation with this kind of $\mathrm{BC}$. The case with inlet $\mathrm{NRBC}$ had to be first run using mass flow inlet $\mathrm{BC}$ so as to match the experimental operating point. It eventually arrives to a steady state, but the initialization process
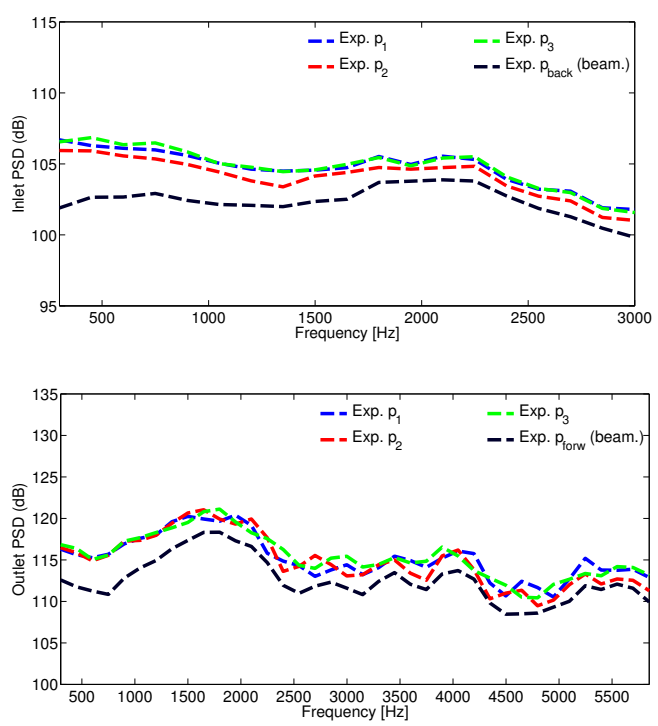

Figure 7: PSD of experimental signals at inlet (top) and outlet (bottom). 

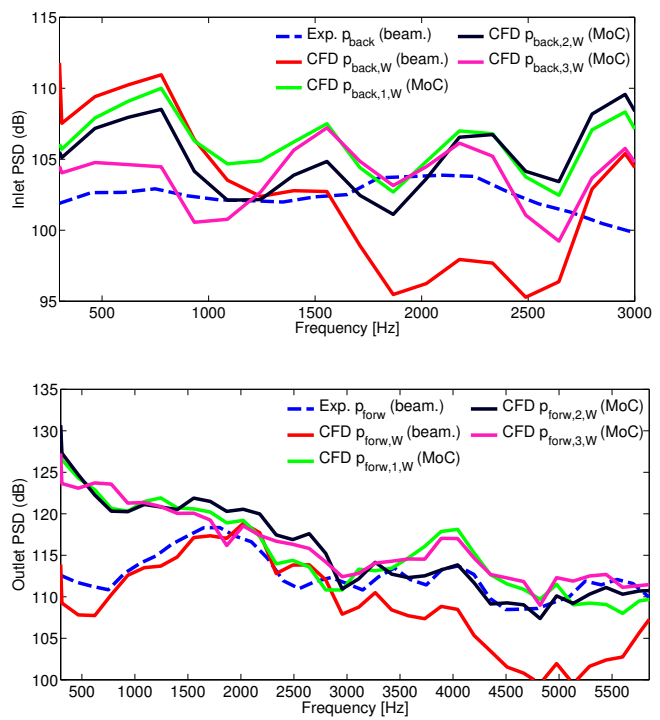

Figure 8: PSD of experimental and numerical probes (wall monitors) at inlet (top) and outlet (bottom).

increases the computational effort. The use of inlet NRBC did not have any impact on outlet duct spectra, but inlet spectra were not similar to those predicted by the case featuring mass flow inlet BC.

One way to overcome the problems derived from avoiding NRBC is to decompose the signals. The relevant pressure components to be analyzed are the ones going out of the compressor wheel (backward pressure at inlet duct and forward pressure at outlet duct). The solution is not exactly the same as with NRBC, since pressure waves are still reflected at the BC, but decomposed signals only include the information going from the compressor to the domain boundaries. Raw pressure spectra of mass flow inlet BC and case with inlet NRBC presented great discrepancies, whereas only slight differences could be noted when pressure components were used instead.

\subsection{Spectra of pressure components}

Figure 7 presents the PSD of experimental pressure signals. The decomposed pressure is obtained using the beamforming technique explained in section 2.3. The counterpart of the decomposition is the reduction of the frequency range that can be analyzed. 
A Nyquist-type criterion based on the spatial resolution of the sensor array is used to exclude aliased high frequencies. The cut-off frequency must be less than half the intrinsic frequency of the array, which corresponds to the wavelength being the separation between sensors [38]:

$$
f_{c}<\frac{f_{i}}{2}=\frac{a}{2 L}
$$

This limit is more restrictive than plane wave cut-off predicted by Eq. 5 . being around $3400 \mathrm{~Hz}$ for the inlet and $6400 \mathrm{~Hz}$ for the outlet, considering their average sound speed and sensor separation. In any case, maximum human hearing sensitivity is comprised in this range.

PSD of three experimental probes in each duct shown at Fig. 7 are quite similar. Decomposed pressure PSD presents a reduction in amplitude regarding raw signals, but no different features are observed. The high coherence between signals is a result of each array of piezoelectric sensor being placed far from the compressor: 6 diameters before and 10 diameters after any crosssection change so that the flow is fully developed. Also, the great length of the ducts avoids any standing wave at frequencies of interest.

In the numerical simulations, the beamforming technique is replicated
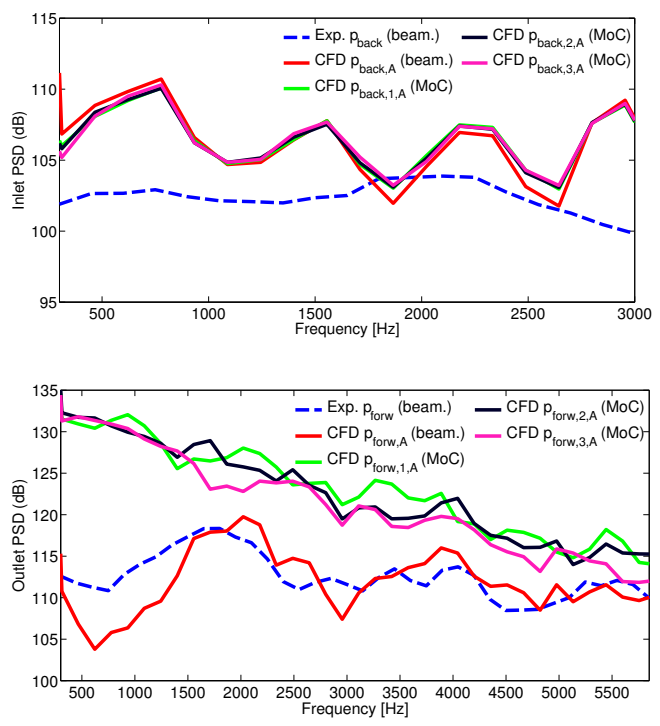

Figure 9: PSD of experimental and numerical probes (axis monitors) at inlet (top) and outlet (bottom) 

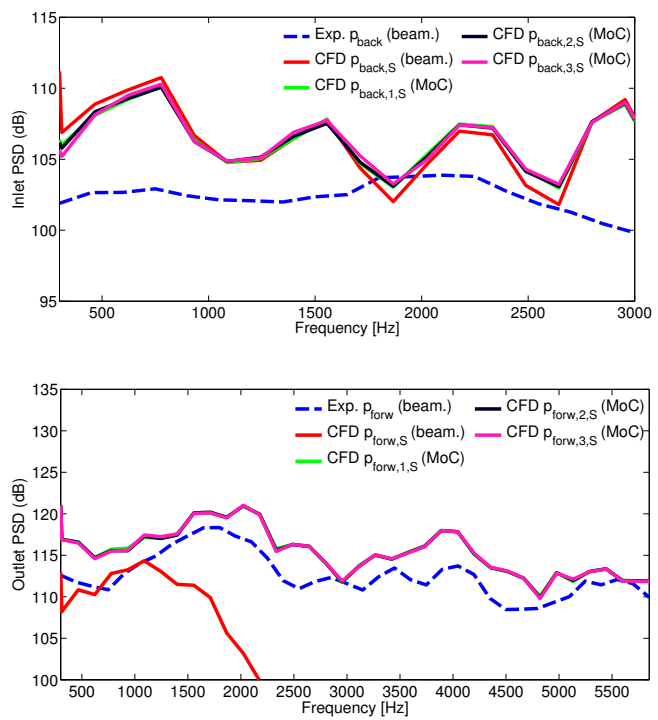

Figure 10: PSD of experimental and numerical probes (cross-section monitors) at inlet (top) and outlet (bottom).

using different types of probes arranged in a 3-array fashion (Fig. 3). Furthermore, the information of the flow field can be used to obtain the pressure components by means of the Method of Characteristics (MoC), as described by Torregrosa et al. [? ]:

$$
\begin{aligned}
& p_{\text {forw }}=p_{\text {ref }}\left[\frac{1}{2}\left(1+\left(\frac{p}{p_{\text {ref }}}\right)^{\frac{\gamma-1}{2 \gamma}}\left(1+\frac{\gamma-1}{2} \frac{u}{a}\right)\right)\right]^{\frac{2 \gamma}{\gamma-1}} \\
& p_{\text {back }}=p_{\text {ref }}\left[\frac{1}{2}\left(1+\left(\frac{p}{p_{\text {ref }}}\right)^{\frac{\gamma-1}{2 \gamma}}\left(1-\frac{\gamma-1}{2} \frac{u}{a}\right)\right)\right]^{\frac{2 \gamma}{\gamma-1}}
\end{aligned}
$$

In Figs. 8, 9 and 10, PSD of the signals obtained using each type of probe in the model are shown. Spectra of pressure components obtained with wall point monitors based on MoC decomposition are slightly dependent on the axial position, both in the inlet and the outlet duct(Fig,8). Moreover, PSD obtained with beamforming is different from those obtained using MoC.

Figure 9 shows that axis monitors provide similar PSD at the inlet duct, regardless of decomposition approach or axial position. Conversely, for these 
virtual sensors, forward pressure spectrum at outlet duct depends on the technique used (beamforming or $\mathrm{MoC}$ ), and MoC based spectra are not coincident.

Inlet spectra obtained with cross-section monitors (Fig. 10) and axis monitors (Fig. 9) are alike: PSD does not depend on probe position when decomposing with Eq. 7 and beamforming spectrum is similar to those obtained with MoC.

In the outlet duct, PSD based on MoC is the same for all cross-section monitors, presenting features close to the ones observed with beamforming technique applied on axis monitors (Fig. 91). However, outlet forward pressure obtained using beamforming technique with cross-section monitors (Fig. 10) provides a spurious spectrum.

Figure 11 is presented to show a snapshot of the compressor flow. For this operating condition, the backflows are attached to the inducer plane and act as an acoustic source, but the flow field in the vicinity of the array is uniform, and both decomposition techniques provide same spectra. In contrast, the flow at the outlet duct is not fully developed and presents a swirling pattern, so the requirements for beamforming technique are not met. In fact, the outlet spectra obtained with beamforming depend on the distance between the same type of virtual sensors (not shown here). This is not a problem for the experimental test rig, since the arrays are placed where the flow is guaranteed to be uniform. Besides, the LCMV weights of the beamforming method are computed so as to have a strong directivity in the axial coordinate, assuming that the signals correspond to aligned points in this direction. When cross-section averages are used, the alignment is not well preserved and beamforming method may provide spurious results (see bottom of Fig. 10).

Regarding $\mathrm{MoC}$ decomposition, the irregular flow in the outlet provides a non-plane forward pressure profile, and thus wall (Fig. 8) and axis probes (Fig. 9) do not provide the same outlet spectra as when the whole crosssection is considered (Fig. 10).

Therefore, the selected comparison between experimental and numerical PSD of pressure component is made using beamforming for obtaining pressure components in the experimental case and Eq. 7) (MoC) at cross section for the numerical simulations. Figure 12 shows this comparison, plotting as well the spectra with the highest frequency resolution attainable by the simulations (about $20 \mathrm{~Hz}$ ) in lighter colors, to highlight the effect of Welch's overlapped segmented average. 
At the inlet duct, experimental PSD is completely flat while numerical spectrum presents bumps centered at $750 \mathrm{~Hz}$ and harmonics. Using a paired difference test, a significant mean difference of $4 \mathrm{~dB}$ is found together with a standard deviation of $2.5 \mathrm{~dB}$.

In the outlet duct, the PSD are about one order of magnitude greater than at the inlet, in accordance with Evans and Ward [1]. A paired difference test between experimental and numerical spectra provides a mean difference of $3 \mathrm{~dB}$ and a standard deviation of $1.8 \mathrm{~dB}$. Spectrum main features are well reproduced by the numerical model. In particular, whoosh noise is present as a broadband elevation in the experimental spectrum between $800 \mathrm{~Hz}$ and $2500 \mathrm{~Hz}$, in agreement with the band of $1-3 \mathrm{kHz}$ used by other researchers [1, 3, 4. Whoosh noise is also captured by the CFD simulation.

\subsection{High frequency spectra}

To avoid nodes of standing waves, pressure decomposition has proved to be a valuable tool in Section 4.3. Nevertheless, the employed beamforming technique sets a constraint in maximum frequency for the comparison between numerical and experimental spectra. Despite human hearing sensitivity peak lies within this range, it would be interesting to extend the validation to higher frequencies.

Observing again Fig. 5, it can be noted that high frequency spectra should be investigated using wall monitors, to take into account higher order modes. Raw pressure spectra could have been used because the effect of standing waves nodes above the onset of first asymmetric mode is not so important,

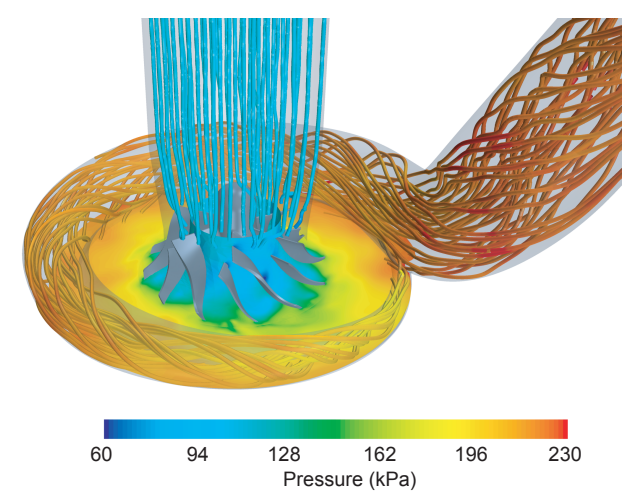

Figure 11: Pressure contours at 50\% rotor span and diffuser combined with flow streamlines at the compressor inlet and outlet, colored by pressure. 

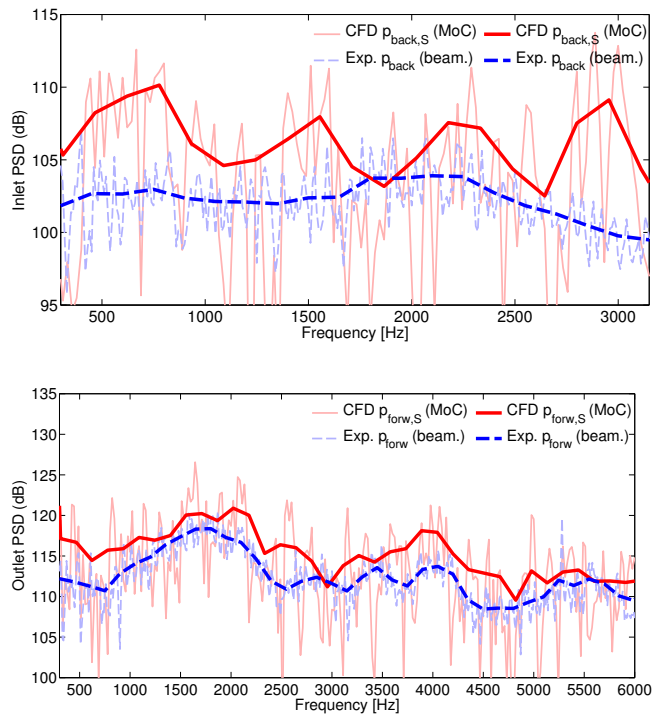

Figure 12: PSD of experimental and numerical pressure components at inlet (top) and outlet (bottom).

since higher order modes prevail over plane waves. However, the impact of probe location and inlet $\mathrm{BC}$ (mass flow or NRBC) in pressure spectra is decreased if pressure components are considered rather than raw pressure in the simulations. In this way, PSD of virtual wall probes can be assessed as a means of validation for frequencies higher than cut-off of asymmetric mode dictated by Eq. 5

Figure 13 shows pressure PSD obtained with each experimental probe. At the inlet, no significant differences are found between spectra of each sensor. Conversely, PSD of the third probe of the array placed at the outlet duct does not show the broadband elevation from 13 to $16 \mathrm{kHz}$ that the other two sensors provide. Taking into account that spectra obtained with the outlet array does only differ in this high-frequency broadband elevation (see Fig. 7), this third sensor might have not been wall-flush mounted during the measurements, providing a different acoustic signature for frequencies above the onset of first asymmetric mode. Hence, experimental spectrum at the outlet duct will be calculated using one of the other two probes, indistinctly.

Figure 14 presents spectra of MoC pressure components (see Eq. 7) obtained with virtual wall sensors, along with experimental spectra of first 
probe. PSD at inlet and outlet ducts is independent of the axial position of the monitors. Moreover, virtual wall sensors at different azimuthal positions provide spectra with no significant differences (not shown here). Thus, wall monitors are a suitable choice to analyze compressor noise generation at frequencies higher than plane wave range.

Numerical inlet and outlet PSD shown at Fig. 14 are similar: an almost constant broadband noise with the only relevant feature of the BPF tone. The inlet broadband elevation from 5 to $12 \mathrm{kHz}$ and subsequent decay observed in the experimental spectrum is not found at numerical PSD. Conversely, the increase in amplitude between 8 and $13 \mathrm{kHz}$ and the constant broadband noise above $16 \mathrm{kHz}$ of the outlet experimental spectrum is accurately predicted by the simulation. The only feature of the outlet spectrum that the model is not able to reproduce is the broadband elevation from 13 to $16 \mathrm{kHz}$.

Throughout all these analysis, outlet numerical spectra are more in agreement with experimental measurements than their inlet counterparts. This could be explained by Fig. 1. Experimental outlet sensor array is placed about $0.4 \mathrm{~m}$ after the domain outlet boundary, whereas this distance extends to $1.4 \mathrm{~m}$ in the inlet duct, including a cross-section change. For high frequencies, mesh spacing should also be considered to explain why outlet spectrum is better predicted. Assuming that at least 20 cells are required to resolve a certain acoustical wavelength [39], mesh density at the outlet duct allows pressure waves with frequencies up to $25 \mathrm{kHz}$ to propagate without numerical damping. At the inlet, this frequency reduces to $12 \mathrm{kHz}$, although BPF tone in Fig. 14 is satisfactorily registered. An example of the effect of numerical damping can be found in the work of Mendonça et al. [8].

\section{Conclusions and future work}

In this paper, a numerical model of a centrifugal compressor has been presented. A detached eddy simulation of an operating condition at peak pressure point predicts global variables (pressure ratio, specific work and isentropic efficiency) with a relative difference less than $1 \%$ in comparison with experimental measurements.

PSD of raw pressure signals obtained with wall, axis and cross-section monitors plotted along maximum human hearing range $(20 \mathrm{~Hz}$ to $20 \mathrm{kHz})$ are coincident only in the plane-wave frequency range. Onset of asymmetric and radial acoustic modes modify the spectra according to the type of monitor. 
Existence of standing waves in the simulation implies that spectra depend also on monitor axial position. Standing waves appear because the modeled domain only includes a fraction of the turbocharger test rig ducts and NRBC are not used because they either require an increase of computational effort or even lead to stability problems.

Pressure components going from the compressor to the boundaries are obtained to mitigate standing waves existing at the CFD model. In the plane wave range, decomposition of pressure signals using the Method of Characteristics with fluid variables calculated at cross-section monitors provides the most consistent signals at the simulation. The arrays of pressure sensors of the steady flow rig are placed so as to guarantee that the flow is fully developed at their position, allowing the use of beamforming for pressure decomposition of experimental signals.

Paired difference test of numerical and experimental PSD calculated in this way shows a significant mean difference of $4 \mathrm{~dB}$ together with a standard deviation of $2.5 \mathrm{~dB}$ at the inlet, whereas the differences are lower for outlet duct spectra.

In the outlet duct, the PSD is about one order of magnitude greater than
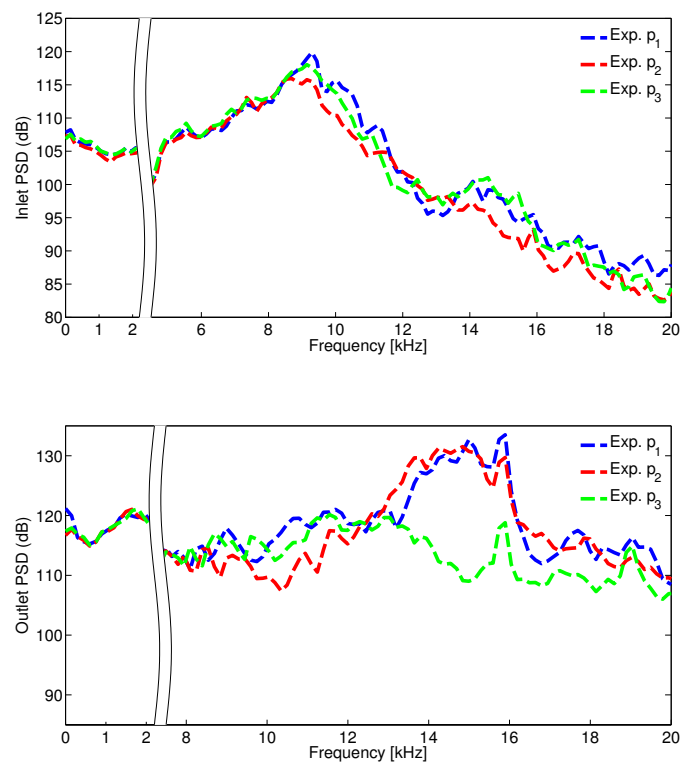

Figure 13: PSD of experimental probes at inlet (top) and outlet (bottom). 

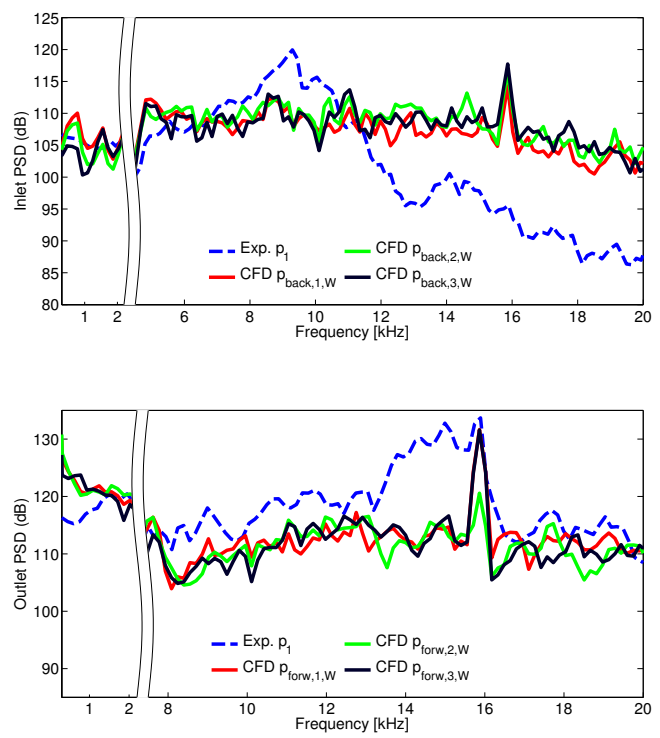

Figure 14: PSD of experimental and numerical wall probes at inlet (top) and outlet (bottom).

at the inlet. Spectrum main features are well reproduced by the numerical model. In particular, the broadband noise existing in the experimental spectum between $1 \mathrm{kHz}$ and $3 \mathrm{kHz}$ is the so-called "whoosh noise", and is captured by the CFD simulation.

For frequencies above plane wave range, virtual wall monitors are used to capture higher order modes. Numerical spectra of pressure decomposed with $\mathrm{MoC}$ fail to predict a decay in amplitude for frequencies above $12 \mathrm{kHz}$ at the inlet duct and a broadband noise between 13 and $16 \mathrm{kHz}$ at the outlet, showing good agreement with experimental ones elsewhere.

Since the CFD simulation is able to detect whoosh noise and global variables along with pressure spectra are in agreement with experimental measurements, the model is a good tool to predict compressor noise generation, according to the validation methodology presented in this paper.

Research is in progress where the numerical model is being used to identify the flow phenomena responsible for whoosh noise. Analysis of acoustic signature of different operating conditions is also being carried out. 


\section{Acknowledgements}

The authors are indebted to the Spanish Ministerio de Economía y Competitividad through Project TRA2012-36954. Part of the computational resources used in this work have been provided by Supercomputing Center of Universitat Politcnica de Valncia and are thus gratefully acknowledged.

\begin{tabular}{lll}
\multicolumn{2}{l}{ List of Symbols } & \\
$a$ & speed of sound & $\mathrm{m} \cdot \mathrm{s}^{-1}$ \\
$c_{p}$ & specific heat capacity at constant pressure & $\mathrm{J} \cdot \mathrm{kg}^{-1} \cdot \mathrm{K}^{-1}$ \\
$D$ & duct diameter & $\mathrm{m}$ \\
$f$ & frequency & $\mathrm{Hz}$ \\
$L$ & sensor separation & $\mathrm{m}$ \\
$\dot{m}$ & mass flow rate & $\mathrm{kg} \cdot \mathrm{s}^{-1}$ \\
$M$ & Mach number & - \\
$N$ & compressor rotational speed & $\mathrm{rpm}$ \\
$p$ & pressure & $\mathrm{Pa}$ \\
$t$ & time & $\mathrm{s}$ \\
$T$ & temperature & $\mathrm{K}$ \\
$u$ & axial velocity & $\mathrm{m} \cdot \mathrm{s}^{-1}$ \\
$\dot{W}$ & compressor absorbed power & $\mathrm{kg} \cdot \mathrm{m}^{2} \cdot \mathrm{s}^{-3}$ \\
$W_{u}$ & compressor specific work & $\mathrm{m} \cdot \mathrm{s}^{-2}$ \\
$\eta_{s}$ & isentropic efficiency & $\%$ \\
$\epsilon_{R}$ & relative error & $\%$ \\
$\gamma$ & ratio of specific heats & - \\
$\phi$ & generic variable & \\
$\Pi_{t, t}$ & total-to-total pressure ratio & - \\
$\tau$ & compressor torque & $\mathrm{kg} \cdot \mathrm{m}^{2} \cdot \mathrm{s}^{-2}$ \\
& &
\end{tabular}




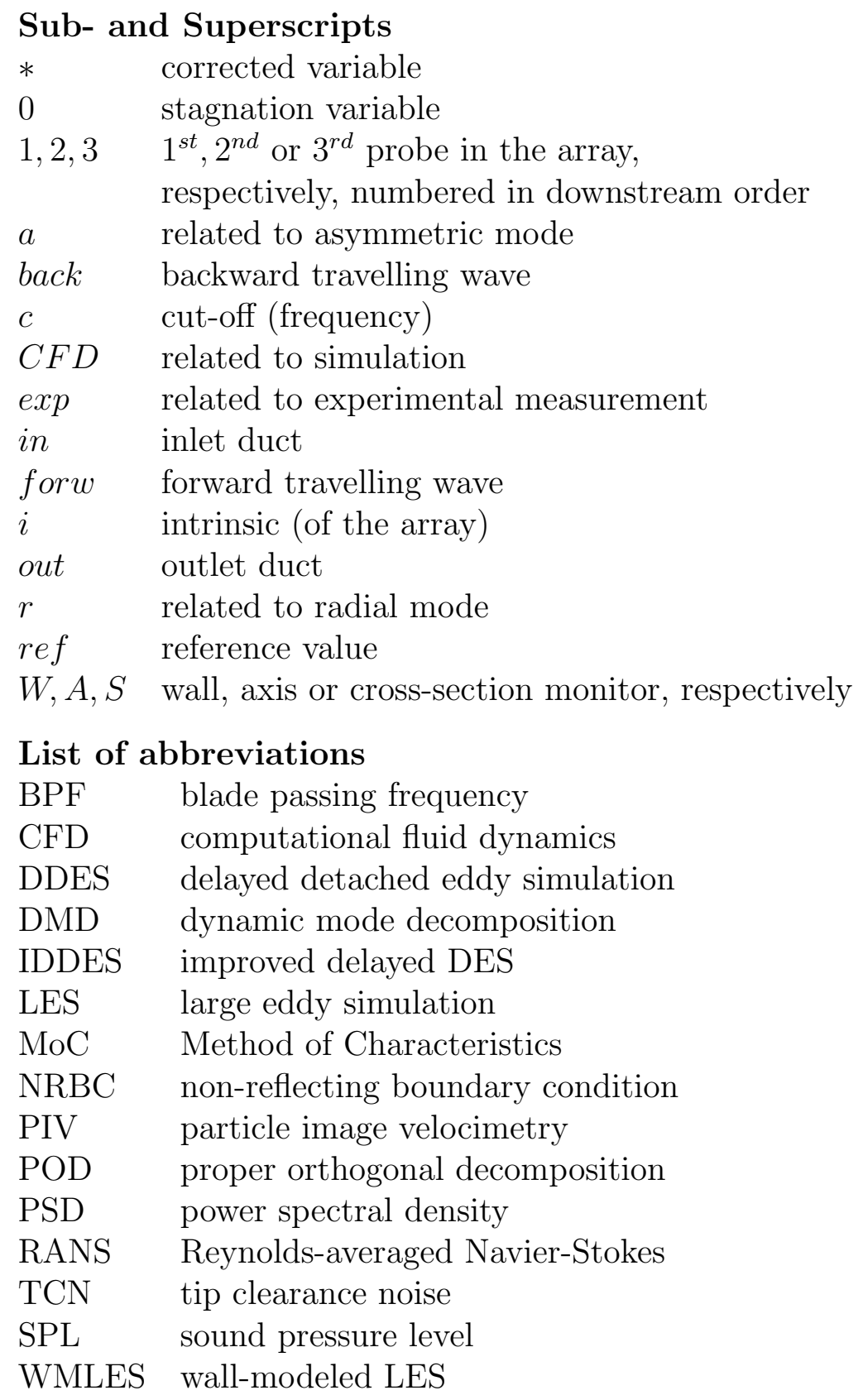

\section{References}

[1] D. Evans, A. Ward, Minimizing Turbocharger Whoosh Noise for Diesel Powertrains, SAE Technical Paper 2005-01-2485. doi:10.4271/ 
2005-01-2485.

[2] C. Teng, S. Homco, Investigation of Compressor Whoosh Noise in Automotive Turbochargers, SAE Int. J. of Passeng. Cars-Mech. Syst. 2 (1) (2009) 1345-1351. doi:10.4271/2009-01-2053.

[3] G. Gaudé, T. Lefèvre, R. Tanna, K. Jin, T. J. B. McKitterick, S. Armenio, Experimental and computational challenges in the quantification of turbocharger vibro-acoustic sources, in: Proceedings of the 37th International Congress and Exposition on Noise Control Engineering (INTERNOISE 2008), Vol. 2008, Institute of Noise Control Engineering, 2008, pp. $5754-5767$.

[4] E. P. Trochon, A new type of silencers for turbocharger noise control, SAE Technical Paper 110 (6) (2001) 1587-1592. doi:10.4271/ 2001-01-1436.

[5] N. Figurella, R. Dehner, A. Selamet, K. Tallio, K. Miazgowicz, R. Wade, Noise at the mid to high flow range of a turbocharger compressor, in: INTER-NOISE and NOISE-CON Congress and Conference Proceedings, Vol. 2012, Institute of Noise Control Engineering, 2012, pp. 8127-8138.

URL http://www.ingentaconnect.com/content/ince/incecp/ 2012/00002012/00000003/art00015

[6] T. Raitor, W. Neise, Sound generation in centrifugal compressors, Journal of Sound and Vibration 314 (2008) 738-756. doi:10.1016/j.jsv. 2008.01 .034 .

[7] C. Sevginer, M. Arslan, N. Sonmez, S. Yilmaz, Investigation of turbocharger related whoosh and air blow noise in a diesel powertrain, in: Proceedings of the 36th International Congress and Exposition on Noise Control Engineering (INTER-NOISE 2007), 2007, pp. 476-485.

[8] F. Mendonça, O. Baris, G. Capon, Simulation of Radial Compressor Aeroacoustics using CFD, in: Proceedings of ASME Turbo Expo 2012, no. GT2012-70028, ASME, 2012, pp. 1823-1832. doi:10.1115/ GT2012-70028.

[9] A. Karim, K. Miazgowicz, B. Lizotte, A. Zouani, Computational aero-acoustics simulation of compressor whoosh noise in automotive 
turbochargers, SAE Technical Paper 2013-01-1880. doi:10.4271/ 2013-01-1880.

[10] Y. Lee, D. Lee, Y. So, D. Chung, Control of Airflow Noise From Diesel Engine Turbocharger, SAE Technical Paper 2011-01-0933. doi: 10.4271/2011-01-0933.

[11] E. Alenius, Flow Duct Acoustics: An LES Approach, Ph.D. thesis, Royal Institute of Technology (KTH) (2012).

[12] A. Sakowitz, M. Mihaescu, L. Fuchs, Flow decomposition methods applied to the flow in an IC engine manifold, Applied Thermal Engineering 65 (1-2) (2014) 57-65. doi:10.1016/j . applthermaleng. 2013.12.082.

[13] A. Kalpakli, R. Örlü, P. Alfredsson, Vortical patterns in turbulent flow downstream a $90^{\circ}$ curved pipe at high womersley numbers, International Journal of Heat and Fluid Flow 44 (2013) 692-699. doi:10.1016/j. ijheatfluidflow.2013.09.008.

[14] J. Galindo, A. Tiseira, P. Fajardo, R. Navarro, Analysis of the influence of different real flow effects on computational fluid dynamics boundary conditions based on the method of characteristics, Mathematical and Computer Modelling 57 (7-8) (2013) 1957-1964, Public Key Services and Infrastructures EUROPKI-2010-Mathematical Modelling in Engineering \& Human Behaviour 2011. doi:10.1016/j.mcm.2012.01.016.

[15] H. Tiikoja, H. Rämmal, M. Abom, H. Boden, Investigations of automotive turbocharger acoustics, SAE International Journal of Engines 4 (2) (2011) 2531-2542. doi:10.4271/2011-24-0221.

[16] G. Piñero, L. Vergara, J. Desantes, A. Broatch, Estimation of velocity fluctuation in internal combustion engine exhaust systems through beamforming techniques, Measurement Science \& Technology 11 (11) (2000) 1585-1595. doi:10.1088/0957-0233/11/11/307.

[17] A. F. Seybert, Two-sensor methods for the measurement of sound intensity and acoustic properties in ducts, The Journal of the Acoustical Society of America 83 (6) (1988) 2233-2239. doi:10.1121/1.396352. 
[18] J. Galindo, J. Serrano, C. Guardiola, C. Cervelló, Surge limit definition in a specific test bench for the characterization of automotive turbochargers, Experimental Thermal and Fluid Science 30 (5) (2006) 449462. doi:10.1016/j. expthermflusci.2005.06.002.

[19] J. Galindo, J. Serrano, X. Margot, A. Tiseira, N. Schorn, H. Kindl, Potential of flow pre-whirl at the compressor inlet of automotive engine turbochargers to enlarge surge margin and overcome packaging limitations, International journal of heat and fluid flow 28 (3) (2007) 374-387. doi:10.1016/j.ijheatfluidflow.2006.06.002.

[20] J. Galindo, F. Arnau, A. Tiseira, R. Lang, H. Lahjaily, T. Gimenes, Measurement and modeling of compressor surge on engine test bench for different intake line configurations, SAE Technical Paper 2011-010370. doi:10.4271/2011-01-0370.

[21] J. R. Serrano, X. Margot, A. Tiseira, L. M. García-Cuevas, Optimization of the inlet air line of an automotive turbocharger, International Journal of Engine Research 14 (1) (2013) 92-104. doi:10.1177/ 1468087412449085 .

[22] J. Galindo, H. Climent, C. Guardiola, A. Tiseira, On the effect of pulsating flow on surge margin of small centrifugal compressors for automotive engines, Experimental Thermal and Fluid Science 33 (8) (2009) 11631171. doi:10.1016/j. expthermflusci.2009.07.006.

[23] CD-adapco, STAR-CCM+, release version 8.04.007 Edition (Juny 2013).

URL http://www.cd-adapco.com

[24] A. Travin, M. Shur, M. Strelets, P. Spalart, Detached-eddy simulations past a circular cylinder, Flow, Turbulence and Combustion 63 (1-4) (2000) 293-313.

[25] M. L. Shur, P. R. Spalart, M. K. Strelets, A. K. Travin, A hybrid RANSLES approach with delayed-DES and wall-modelled LES capabilities, International Journal of Heat and Fluid Flow 29 (6) (2008) 1638-1649. doi:10.1016/j.ijheatfluidflow.2008.07.001.

[26] J. R. Serrano, P. Olmeda, F. Arnau, M. Reyes-Belmonte, A. Lefebvre, Importance of Heat Transfer Phenomena in Small Turbochargers for 
Passenger Car Applications, SAE International Journal of Engines 6 (2) (2013) 716-728. doi:10.4271/2013-01-0576.

[27] J. R. Serrano, F. J. Arnau, R. Novella, M. Á. Reyes-Belmonte, A procedure to achieve 1d predictive modeling of turbochargers under hot and pulsating flow conditions at the turbine inlet, SAE Technical Paper 2014-01-1080 (2014) 13pp. doi:10.4271/2014-01-1080.

[28] J. Serrano, P. Olmeda, F. J. Arnau, A. Dombrovsky, L. Smith, Methodology to characterize heat transfer phenomena in small automotive turbochargers: experiments and modelling based analysis, in: Proceedings of ASME Turbo Expo 2014, 2014.

[29] A. Hemidi, F. Henry, S. Leclaire, J.-M. Seynhaeve, Y. Bartosiewicz, CFD analysis of a supersonic air ejector. Part I: experimental validation of single-phase and two-phase operation, Applied Thermal Engineering 29 (8-9) (2009) 1523-1531. doi:10.1016/j.applthermaleng.2008.07. 003.

[30] A. Hemidi, F. Henry, S. Leclaire, J.-M. Seynhaeve, Y. Bartosiewicz, CFD analysis of a supersonic air ejector. Part II: Relation between global operation and local flow features, Applied Thermal Engineering 29 (1415) (2009) 2990-2998. doi:10.1016/j .applthermaleng. 2009.03.019.

[31] M. Ubaldi, P. Zunino, G. Barigozzi, A. Cattanei, An experimental investigation of stator induced unsteadiness on centrifugal impeller outflow, Journal of turbomachinery 118 (1996) 41-51. doi:10.1115/1.2836604.

[32] F. Hellström, E. Guillou, M. Gancedo, R. DiMicco, A. Mohamed, E. Gutmark, L. Fuchs, Stall Development in a Ported Shroud Compressor using PIV Measurements and Large Eddy Simulation, Tech. rep., SAE Technical Paper 2010-01-0184 (2010). doi:10.4271/ 2010-01-0184.

[33] B. Semlitsch, V. JyothishKumar, M. Mihaescu, L. Fuchs, E. Gutmark, M. Gancedo, Numerical Flow Analysis of a Centrifugal Compressor with Ported and without Ported Shroud, SAE Technical Paper 2014-01-1655. doi:10.4271/2014-01-1655. 
[34] B. W. van Oudheusden, Piv-based pressure measurement, Measurement Science and Technology 24 (10) (2013) 32pp. doi:10.1088/0957-0233/ 21/10/105401.

[35] M. Choi, N. H. Smith, M. Vahdati, Validation of Numerical Simulation for Rotating Stall in a Transonic Fan, Journal of turbomachinery 135 (2). doi:10.1115/1.4006641.

[36] P. Welch, The use of fast fourier transform for the estimation of power spectra: a method based on time averaging over short, modified periodograms, Audio and Electroacoustics, IEEE Transactions on 15 (2) (1967) 70-73.

[37] L. J. Eriksson, Higher order mode effects in circular ducts and expansion chambers, The Journal of the Acoustical Society of America 68 (1980) 545. doi:10.1121/1.384768.

[38] M. Åbom, H. Bodén, Error analysis of two-microphone measurements in ducts with flow, The Journal of the Acoustical Society of America 83 (6) (1988) 2429-2438. doi:10.1121/1.396322.

[39] F. Mendonça, A. Read, F. Imada, V. Girardi, Efficient CFD Simulation Process for Aeroacoustic Driven Design, SAE Technical Paper 2010-360545. doi:10.4271/2010-36-0545. 